\title{
Las razones para redefinir la muerte y su crítica*
}

\author{
Alberto Cuauthémoc Mayorga Madrigala - Dalila Ayala Castillob
}

\begin{abstract}
Resumen: A más de cincuenta años del Informe de Harvard, la redefinición propuesta para la muerte continúa siendo problemática y no goza de un consenso internacional. La posibilidad de trasplantar órganos vitales y el avance de los soportes artificiales para el mantenimiento de algunas funciones sustantivas impulsó la necesidad de redefinir la noción de muerte. Al proponer nuevas definiciones surge también un conjunto de interrogantes éticos y técnicos. En este texto exponemos algunas de las principales razones y críticas a la pretensión de definir la muerte encefálica como muerte. Para dicho propósito, realizamos un análisis de las críticas que, sobre este punto, hizo Hans Jonas, así como las refutaciones a su posición. Por otra parte, presentamos algunos de los criterios en que se fundamentan las pruebas diagnósticas y la escasez del consenso en este sentido. De esta manera pretendemos mostrar que los diferentes puntos de vista para definir la muerte, a pesar de no lograr acuerdos entre las diferentes comunidades, requieren un análisis y debate continuos con el fin de dar atención sustentada a los factores que motivaron la redefinición de la muerte.
\end{abstract}

Palabras clave: muerte; muerte encefálica; vida; ética; diagnóstico

Recibido: 20/07/2020 Aceptado: 30/11/2020

Disponible en línea: 23/07/2021

Cómo citar: Mayorga Madrigal AC, Ayala Castillo D. Las razones para redefinir la muerte y su crítica. Rev. latinoam. bioet [Internet]. 23 de julio de 2021 [citado 23 de julio de 2021];21(1):61-76. Disponible en: https://revistas.unimilitar.edu.co/index.php/rlbi/article/view/5018

* Artículo de investigación apoyado por el Centro Universitario de Ciencias Sociales y Humanidades de la Universidad de Guadalajara a través del programa de fomento de la investigación 7.1-2018 al proyecto ¿Cómo argumentar en bioética?

a Doctor en Filosofía. Universidad de Guadalajara, Guadalajara, Jalisco, México.

Correo electrónico: c_mayorgam@hotmail.com

ORCID: https://orcid.org/0000-0002-4552-5754

b Maestra en Estudios filosóficos. Universidad TecMilenio, Zapopan, Jalisco, México.

Correo electrónico: dalila_ayala@tecmilenio.mx

ORCID: https://orcid.org/0000-0002-0808-3328 


\section{Reasons to Define Death and its Criticism}

Abstract: Over fifty years from Harvard's report, the redefinition proposed for death still poses problems and has not reached international consensus. The possibility to transplant vital organs and the progress of artificial support to maintain some substantive functions raised the need to redefine the notion of death. Proposing new definitions also gives place to new ethical and technical questions. In this context we present some of the main reasons and criticism to the intention to define death as encephalic death. For such reasons we analyze some of the criticism made on this point by Hans Jonas, as well as challenges to his position. On the other hand, we present some of the criteria diagnostic tests are based on, and the lack of consensus in this regard. With his analysis we aim at showing that the different viewpoints to define death, despite not reaching agreement among different communities, require continued analysis and debate in order to give supported attention to the factors motivating the redefinition of death.

Keywords: death; encephalic death; life; ethics; diagnostics.

\section{Razões para redefinir a morte e sua crítica}

Resumo: Há mais de 50 anos do Relatório da Harvard, a redefinição proposta para a morte continua sendo problemática e não usufrui de um consenso internacional. A possibilidade de transplantar órgãos vitais e o avanço dos recursos artificiais para manter algumas funções substanciais impulsionou a necessidade de redefinir a noção de morte. Ao propor novas definições, surge também um conjunto de questionamentos éticos e técnicos. Neste texto, expomos algumas das principais razões e críticas da pretensão de definir a morte encefálica como morte. Para isso, realizamos uma análise das críticas que, desse ponto, Hans Jonas fez, bem como as refutações a suas posições. Por sua vez, apresentamos alguns dos critérios nos quais os exames diagnósticos e a escassez do consenso nesse sentido são fundamentados. Com esta análise, pretendemos mostrar que os diferentes pontos de vista para definir a morte, apesar de não chegarem a acordos entre as comunidades, requerem análise e debate contínuos a fim de dar atenção especial aos fatores que motivaram a redefinição da morte.

Palavras-chave: morte; morte encefálica; vida; ética; diagnóstico 


\section{Introducción}

Con el fin de comprender las posturas en torno a la definición de muerte encefálica, presentamos algunos de los argumentos más influyentes que se han aportado a la discusión para la creación y desarrollo de su definición y diagnóstico. Para ello, pretendemos clarificar el panorama vigente de la discusión sobre la definición, analizando primero la postura del filósofo Hans Jonas quien, en su artículo "Against Stream" (16), se opuso a definir la muerte encefálica como muerte y, como consecuencia, sostiene que una "muerte encefálica" no nos autoriza a hacer uso de los órganos con fines de trasplante, dado que no podemos afirmar que la persona esté muerta de hecho, puesto que no conocemos los límites entre la vida y la muerte. Además, sugiere que no se deberían extraer los órganos de un individuo declarado con muerte encefálica al no saber si con ello se estaría causando su muerte. Por otra parte, la referencia a los trasplantes de órganos resulta significativa para la comprensión de la muerte encefálica ya que son los individuos diagnosticados de esta manera los candidatos óptimos para la donación cadavérica. A propósito, más adelante revisaremos la ablación de los órganos para trasplantes como una de las razones destacadas en el Informe Harvard para definir la muerte encefálica. Aunque la objeción de Hans Jonas tiene más de 40 años, todavía genera interrogantes relevantes a la discusión en la búsqueda de consensos.

Por lo tanto, es necesario analizar los criterios utilizados para el diagnóstico de la muerte encefálica en el Informe de Harvard y en algunas modificaciones por las comisiones correspondientes hasta llegar al estado actual de dichos criterios en algunas legislaciones internacionales, revisadas en: el informe sobre Muerte encefálica en Iberoamérica (9) de la Red/Consejo Iberoamericano de Donación y trasplantes y en las Conclusiones de la III Conferencia de Consenso de la Sociedad Española de Medicina Intensiva, Crítica y Unidades Coronarias (semicyuc), Muerte encefálica en las Unidades de Cuidados Intensivos (7). Tanto el Informe de la Red/Consejo Iberoamericano como las conclusiones de la SEMICYUC son analizados por sus respectivos objetivos: el primero busca revisar si existe uniformidad en los criterios diagnósticos fundamentales de la muerte encefálica en la práctica médica; por su parte, el segundo tiene como objetivo llegar a un correcto consenso, por parte de expertos, sobre los criterios para el diagnóstico de la muerte encefálica.

El problema a lo largo de la historia en el diagnóstico de muerte radica en que, al aplicar los métodos requeridos para constatarla, se han presentado errores relacionados con el hecho de declarar muerta a una persona que aún se encuentra con signos vitales. Cabe aclarar que no hablamos de errores derivados de un mal procedimiento en el diagnóstico, sino que tales errores, los cuales llamaremos "falsos positivos", se dan debido a que los métodos para diagnosticar fallecimiento son insuficientes para abarcar todo el fenómeno de la muerte. Lo anterior también se asocia al problema de la noción de muerte, lo que nos produce incertidumbres sobre nuestras creencias acerca de la muerte y nos lleva a preguntarnos: ¿cómo saber cuándo una persona está muerta? y ¿qué es la muerte? Al respecto, para Platón la muerte ocurría cuando el alma se separaba del cuerpo porque este último solo era un recipiente para el alma mientras estaba en la Tierra; en otras palabras, moría solo el cuerpo del individuo (28). Estos dos problemas están asociados porque a lo largo de la historia se ha entendido la muerte como la pérdida de la vitalidad del cuerpo y el fin de la existencia personal, por tanto, la noción de Platón afirma que en realidad lo que muere es el recipiente, mientras que el alma, donde reside la existencia del yo, sigue viviendo. En cambio, para su discípulo Aristóteles la vida no residía solo en el alma, sino que la vida se daba en una combinación integral de alma y cuerpo. El alma no puede existir sin el cuerpo y, por lo tanto, la muerte del cuerpo implica la muerte del alma (30). Las anteriores nociones nos llevan a otra cuestión importante sobre la relación que se da entre la noción y diagnóstico de muerte: al establecer los criterios para diagnosticar la muerte, ¿podemos configurar nuestra noción de muerte? o ¿la noción de muerte precede y fundamenta los criterios de diagnóstico?

Las primeras observaciones acerca de la muerte se dieron entre carniceros, cirujanos, guerreros y verdugos, quienes concluían que las partes del 
cuerpo no siempre morían al mismo tiempo. Los médicos griegos pensaban que la muerte podía darse en los pulmones, el cerebro o el corazón, pero que el único que consideraban sede de la vida era el corazón. Galeno, Hipócrates y los anatomistas de Alejandría le daban al cerebro un papel vital, ya que en él se encontraba la sensación, el movimiento y la razón (30). Podemos darnos cuenta de que estas afirmaciones solo aportaban a la definición o noción de muerte, pero no al diagnóstico de la muerte. Además, tal problema no era propiamente de los médicos:

Así, cuando los médicos clásicos y medievales hablaban de las propiedades post-mortem, los "signos de la muerte", no mencionaban los latidos del corazón, el pulso o la respiración, sino que repetían el retrato de la muerte inminente pintado en el Prognostikon de Hipócrates. Entonces, como ahora, los "signos de muerte" indicaban cuándo había terminado el trabajo del médico, pero para los médicos clásicos estos "signos" no significaban que el paciente estuviera realmente muerto. Durante la mayor parte de la historia occidental, el diagnóstico real de muerte fue principalmente una función no médica $(30, \text { p20 })^{1}$.

Estos signos de muerte daban cuenta del alcance de la labor del médico. No obstante, el paciente podría encontrarse vivo sin siquiera saberlo.

Ya en el siglo XVII, Antoine Louis afirmó que el inicio del rigor mortis era un signo de diagnóstico suficiente. No obstante, en este mismo siglo William Harvey describió la circulación y estableció el latido cardíaco como signo de vida. Posteriormente, Laënnec (1781-1826) inventó el estetoscopio en 1819 y logró así escuchar los latidos del corazón y apoyar al diagnóstico de muerte basado en este órgano. Sin embargo, seguían existiendo imprecisiones en el diagnóstico y los falsos positivos continuaban apareciendo. Como consecuencia, en los

1 Thus, when classical and medieval physicians spoke of the proprietates mortis, the "signs of death," they did not mention heartbeats, pulse, or breath, but repeated the portrait of impending death painted in Hippocrates' Prognostikon. Then as now, the "signs of death" indicated when the doctor's job was finished, but for classical physicians these "signs" did not mean the patient was actually dead. For most of Western history, the actual diagnosis of death was primarily a non-medical function. siguientes siglos se cometieron muchos errores al diagnosticar la muerte: El médico parisiense Jean Bruhier recopiló los relatos correspondientes a veinticinco personas que supuestamente fueron enterradas vivas, y de setenta y dos cuya muerte se certificó por error. En el siglo XIX se registraron cuarenta y seis casos de personas que volvieron a la vida mientras esperaban ser inhumadas $(15, \mathrm{p} 363)$.

Por su parte, en el siglo XIX basaban los procedimientos para diagnosticar la muerte en el criterio cardio-respiratorio; para ello, colocaban un espejo frente a la nariz del paciente con el fin de verificar la ausencia de respiración, también abrían una arteria para comprobar ausencia de circulación e incluso introducían la cabeza del paciente en agua para comprobar si exhalaba burbujas. Estos métodos fueron apoyados con la creación del estetoscopio (42). Sin embargo, aún existían imprecisiones en la definición: La muerte es el cese de un fenómeno con el que estamos singularmente muy familiarizados, el fenómeno de la vida. Esta definición es citada en Los principios de medicina forense (15). Por otro lado, existían factores que podían falsificar la muerte como el frío extremo, los opiáceos, el alcohol, las hemorragias, la apoplejía, la asfixia, fiebre alta y lesión en la cabeza.

En 1788 Charles Kite de London Humane Society afirmó que la falta de contracción, en respuesta a la reanimación eléctrica, era útil como prueba para constatar la pérdida de la función neuromuscular. Esta desconfianza se mantiene durante el siglo XIX y se llega a afirmar que el único signo real para constatar la muerte es el signo de la putrefacción, pero incluso algunos científicos ponen en duda esto: La desconfianza que se tenía respecto de los diagnósticos de muerte se mantiene hasta el siglo XIX, época en la que el mismo Dunglison afirma que "el único signo seguro de muerte real es el comienzo de la putrefacción", mientras que otros, como W. Hawes o M.J.B. Orfilia, dudan incluso de que sea una prueba suficiente (42).

Gracias a la importancia que adquiere la tecnología en las cuestiones médicas, podemos afirmar que desde el siglo XX se dieron grandes cambios en la noción de muerte:

Los países occidentales han visto, sobre todo a partir de los años cincuenta, cómo la tecnología aplicada al 
soporte vital (respiradores automáticos, reanimación cardio-respiratoria, corazones artificiales) ha dotado a la medicina de la capacidad de mantener a una persona con vida durante periodos de tiempo que hasta entonces eran inconcebibles (42).

Por esta razón, pretendemos responder las siguientes cuestiones: ¿cuál es el estado actual de la definición de muerte encefálica? y ¿con qué criterios podemos afirmar que el individuo con muerte encefálica está realmente muerto? La importancia de poder contestar estas preguntas actualmente nos dará luz acerca de las implicaciones prácticas en la muerte encefálica, tales como: la decisión de abstenerse a todo tratamiento terapéutico o de retirar el soporte vital, así como la decisión de extraer los órganos con fines de trasplante.

\section{Hans Jonas frente a la redefinición de la muerte}

El Informe de Harvard menciona a los individuos cuya condición clínica conduce a la necesidad de redefinir la muerte. Dicho comité afirmó que el coma irreversible tiene muchas causas, pero solo están interesados en "aquellos individuos en coma que no tienen actividad discernible en el sistema nervioso central" (37, p337). En este sentido, se exponen dos razones por las cuales se justifica ética y socialmente una nueva definición de muerte basada en los siguientes criterios neurológicos: por un lado, los pacientes con el corazón latiendo y el cerebro irreversiblemente dañado suponen una carga grande para sus familiares, hospitales y para aquellos que necesitan los recursos de los cuales estos hacen uso; por otro lado, los criterios obsoletos para definir la muerte pueden generar controversias en la obtención de órganos para trasplantes (37, p337).

En un análisis de las razones prácticas para redefinir la muerte, Hans Jonas pretende especificar los fundamentos que motivan al Comité de Harvard para ofrecer esta nueva definición: primero, liberar a pacientes, allegados y recursos médicos de las cargas de un coma prolongado indefinidamente; segundo, evitar las controversias sobre la obtención de órganos para trasplante; y tercero, el médico tiene el derecho de finalizar el tratamiento de un estado cuya prolongación no tiene sentido para el paciente porque no implica una mejoría (16, p148). Para Jonas, la tercera razón es el único fundamento válido para afirmar la necesidad de la definición, ya que la prolongación del estado vegetativo en las condiciones señaladas no tiene sentido (aquí se atienden también algunos aspectos relativos a la primera razón). De llegar a considerar lo anterior, se estaría liberando al paciente y, por consiguiente, a los allegados $y$, adicionalmente, habría una optimización de recursos médicos. Por lo tanto, Jonas está de acuerdo con el Comité de Harvard en que no tiene sentido una existencia meramente vegetativa. En cuanto a la segunda razón sobre la libertad para la utilización de órganos, esta no recae en el beneficio del paciente en sentido estricto, sino en la búsqueda del beneficio de otros. Sin embargo, Jonas expresa que con dicha propuesta el Comité de Harvard no ha definido en estricto sentido la muerte, sino que solo ha dado un criterio para admitir, sin obstáculos, la muerte y permitir la desconexión del respirador artificial ante la existencia vegetativa del individuo en coma irreversible. Si bien este criterio es la única razón válida para que la muerte sea reconocida, eso no significa que tal criterio sea la definición de muerte ni el único sustento que da derecho al médico de darle fin a la vida del paciente.

Para postular la definición y el criterio de muerte, es necesario dar respuestas a cuestiones ontológicas y epistemológicas como: ¿qué es la muerte? y ¿cómo sabemos que alguien ha muerto? Según Rivera, la primera pregunta es una convención lingüística que no es verdadera ni falsa, sino adecuada o inadecuada; por su parte, la segunda pregunta se responde con los criterios de muerte y es principalmente empírica (27, p35).

Jonas critica, en primera instancia, la afirmación de que el coma irreversible debe reconocerse como una nueva definición de muerte, entendiendo el coma irreversible como la ausencia de actividad discernible en el sistema nervioso central. Él no está en contra de desconectar el respirador a personas en coma irreversible, sino en contra de asegurar que las personas en coma irreversible están muertas de hecho, justificar suspensión del respirador artificial y, por consiguiente, admitir la extracción de órganos con fines de trasplante. 
Teniendo en cuenta el desconocimiento de la delimitación entre la vida y la muerte y, por consiguiente, la falta de certeza sobre si alguien diagnosticado con coma irreversible está muerto de hecho, el acto de no detener el respirador va en contra de su derecho a morir, pero, por otro lado, el acto de extraer los órganos podría estar causando la muerte del paciente. Si aceptamos que el hecho de que estén muertos nos da el derecho a extraer sus órganos, entonces justificaríamos otras prácticas como hacer uso de los cuerpos como fábrica de hormonas y otras sustancias bioquímicas, utilizarlos como bancos de sangre o incluso hacer uso de estos con fines de experimentación en trasplantes $\mathrm{u}$ otras investigaciones (16, p153).

Para hablar sobre el derecho a morir, Hans Jonas distingue entre el sujeto activo que puede decidir el suicidio y el paciente mortalmente enfermo que está expuesto pasivamente a las técnicas de retraso de la muerte de la medicina moderna; tal distinción nos lleva a otra: dejar morir y causar la muerte (16, p160). El hecho de dejar morir o permitir que el paciente muera se vuelve una obligación del médico para proteger a la persona. Se refiere al derecho de morir como la libertad de elegir morir cuando la vida no es digna de ser vivida. En el caso de las personas en coma irreversible, apela a la protección de la persona que el paciente fue un día y cuya memoria se ve disminuida por la degradación de tal pervivencia $(16, \mathrm{p} 171)$.

La posición de Jonas nos lleva a la segunda crítica respecto a las implicaciones éticas que conlleva aceptar que el individuo en coma irreversible es un cadáver y no un paciente. Lo anterior tiene que ver con la segunda razón práctica que declara el Comité de Harvard en relación con la necesidad de reformular los criterios de muerte para evitar la controversia en la obtención de órganos para trasplantes. Jonas afirma que si admitimos que podemos extraer los órganos del paciente solo con certificar que el individuo está muerto, también podría justificarse la utilización del cuerpo para otros fines de experimentación u otras investigaciones como banco de sangre, de hormonas o sustancias químicas; lo cual sería inaceptable.

\section{Tres réplicas al punto de vista}

\section{de Hans Jonas}

Las siguientes críticas a la postura de Hans Jonas son resultado de un intercambio de ideas de manera informal entre el cirujano Samuel Kountz, el psiquiatra Harryson Sadler, el historiador de la medicina Otto Guttentag y Hans Jonas. Aquí sintetizamos las tres objeciones a la posición de Jonas y la réplica:

1) Su argumento sobre los cadáveres donantes impediría serios esfuerzos médicos por salvar vidas. A esta crítica, Jonas responde que se comete una petición de principio, es decir, que la proposición que se intenta establecer se supone involuntariamente al afirmar que el estado del individuo en coma irreversible es de un cadáver donante, pues precisamente el estado de cadáver está en cuestión.

2) Explicar hechos científicos con vagas consideraciones filosóficas. A esta segunda crítica, responde que sí se presenta dicha vaguedad, pero que esta podría implicar que efectivamente estamos ante un fenómeno que es en sí mismo vago. Al hablar del límite entre la vida y la muerte, estamos hablando de un estado incierto, pues, según Jonas, no conocemos el límite entre la vida y la muerte.

3) Desconocía la diferencia entre "muerte del organismo como un todo" y muerte de "todo el organismo", a la vez que la diferencia entre respiración espontánea e inducida desde fuera $y$ resto del movimiento del cuerpo. Frente a la tercera objeción, afirma que está claro que no es lo mismo la muerte de todo el organismo que la muerte del organismo como un todo, pero aclara que él siempre se ha referido a la muerte del organismo como un todo y, ya que la circulación sanguínea y la respiración son actividades que engloban todo el sistema, debe considerarse la muerte del organismo al presentarse el cese de estas funciones. Sin embargo, en este último punto introduce la diferencia entre un activador interno (el sistema nervioso central) y un activador externo (el respirador artificial); con esto explica que un cese puede ser irreversible en cuanto a su espontaneidad, pero aún 
reversible en cuanto a la actividad misma. Entonces, si hay un activador externo, el cese de la actividad misma es reversible, pero al estar dañado el sistema nervioso central irreversiblemente, la espontaneidad también es irreversible. La importancia de la distinción que hace Jonas entre la irreversibilidad de la actividad y la irreversibilidad de la espontaneidad nos permite reconocer que si tuviéramos la oportunidad de activar externamente el cerebelo, lo haríamos; con esto un médico no podría afirmar que un individuo está muerto solo por el hecho de no tener actividad espontánea en su cerebelo, pues, a pesar de ello, la actividad continúa. En el siguiente fragmento Jonas argumenta con mayor claridad su objetivo al enfatizar en la espontaneidad de la actividad:

En el coma irreversible, tal como lo definía el grupo de Harvard, el punto de partida es naturalmente un estado que excluye la reactivación de cualquier parte del cerebro en todos los sentidos. El cerebro, tenemos que decir entonces, está muerto. Tenemos entonces a un organismo como un todo, menos el cerebro mantenido en un estado de vida parcial mientras el respirador y otros auxiliares estén funcionando (16, p152).

Siguiendo el argumento anterior, se afirma que mientras haya actividad en los sistemas circulatorio y respiratorio (mediante un activador externo), el organismo como un todo sigue vivo; lo que está muerto es el cerebro (el activador interno encargado de la espontaneidad de la actividad). No obstante, el Comité de Harvard no estaría de acuerdo con afirmar que tenemos entonces a un organismo como un todo menos el cerebro; en cambio, sostiene que si el cerebro pierde sus funciones y no tiene posibilidad de volver a funcionar, el individuo está muerto para todo propósito práctico, entre estas funciones menciona la espontaneidad para respirar. Además, como Jonas lo expresa, su razonamiento sobre la espontaneidad se basa en un ejemplo hipotético sobre la reactivación externa del cerebelo. Como resultado del razonamiento de Jonas, cabe preguntarnos qué debemos hacer con el paciente. La importancia de esta cuestión no radica propiamente en la búsqueda de una respuesta sobre los hechos, sino en la búsqueda de cursos de acción, es decir, se dirige a un problema de naturaleza práctico, a la valoración de hechos bajo incertidumbre sobre los cuales hay que tomar decisiones.

En conclusión, Jonas está de acuerdo con la tercera razón práctica que expresa el Comité de Harvard sobre la falta de sentido de una vida vegetativa, la cual figura como la única razón válida para desconectar el respirador y detener otros medios que mantienen en este estado al individuo (16, p149). Es correcto dejar morir al paciente, pero no hay ninguna razón para extraer los órganos para trasplante de individuos en coma irreversible porque se podría estar causando su muerte. Cabe mencionar esta posibilidad, ya que, como desconocemos el límite entre la vida y la muerte, tampoco sabemos si la persona en coma irreversible ha muerto o aún se encuentra con vida. Por otro lado, recordemos que el hecho de no permitir que el paciente muera va contra su derecho a morir y el consentir que se extraigan los órganos para trasplante mediante la justificación de que ya es un cadáver abre las puertas a prácticas cuestionables.

Una de las críticas más recientes a la postura de Jonas es presentada por F. G. Miller en su artículo "Death and organ donation", donde afirma estar de acuerdo con Jonas en que los pacientes con muerte encefálica no parecen ser ni son cadáveres. Considera que actualmente tal conclusión es más evidente y certera debido a los avances en las investigaciones acerca del funcionamiento biológico y la muerte encefálica (19, p617). Sin embargo, Miller pregunta si es incorrecto extraer los órganos vitales de estos pacientes y su posición consiste en que la persona con muerte encefálica no está muerta de hecho y que no hay explicación plausible para afirmar lo contrario. Para él, los argumentos más consistentes de Jonas ante la práctica de la extracción de órganos para trasplante son los siguientes:

1) No podemos estar seguros de que estos seres humanos que siguen respirando y con el corazón latiendo sean inmunes al sufrimiento.

2) Es una explotación injusta extraer órganos vitales de pacientes vivos sin importar cuán neurológicamente comprometidos estén.

3) Para los médicos, realizar la práctica de 
donación de órganos letales está en contra de la vocación moral de la medicina. $(19$, p617)

Miller responde al primer argumento, señalando que el estado de los individuos con muerte encefálica no permitiría sentir dolor y, si así fuera, podría resolverse con anestesia. Al segundo argumento, responde que seguramente Jonas no habría puesto objeción a la obtención de órganos de personas muertas de hecho siempre y cuando se cuente con el consentimiento del paciente (19, p617). Al respecto, sostiene que, aunque el paciente no haya expresado el consentimiento, sí pudo haber manifestado sus preferencias con anterioridad o los familiares podrían tomar la decisión en su nombre (19, p618). Sin embargo, el problema es aun más complicado dado que tanto Miller como Jonas están de acuerdo al sostener que el individuo con muerte encefálica parece no estar muerto, en tal caso, el consentimiento se daría para la extracción de los órganos vitales de un paciente en coma irreversible; no obstante, queda en duda si aún está vivo. Por tanto, esta es la cuestión ética a considerar: ¿es legítima la extracción de órganos vitales a un paciente en coma irreversible (no cadáver) con fines de trasplante, cuyo procedimiento implica la muerte del paciente? Miller responde que si aceptamos que es legítimo detener el soporte vital para que se "permita" que el individuo muera, también debe ser legítimo el acto de extracción de órganos vitales de pacientes en coma irreversible, porque considera que ambos actos causan la muerte del individuo.

Ante la premisa que afirma que al detener el soporte vital se ocasiona la muerte, Miller objeta que permitir la muerte implique pasividad en el proceso de morir, puesto que tal permisibilidad es la causa de la muerte. Permitir que el paciente muera es una "ficción moral", es decir, una creencia falsa motivada moralmente, respaldada para preservar la validez absoluta de aquella norma tradicional que señala que los doctores no deben matar a los pacientes $(19, \mathrm{p} 618)$. Por consiguiente, con el acto de detener el soporte vital causamos la muerte, si esto es así y este acto es legítimo, Miller no ve por qué se haría algún mal al paciente al extraer sus órganos, dado que también sería legítimo en la medida en que ninguno de los dos actos es pasivo.

Sin embargo, posteriormente Miller recurre de nuevo a la ficción moral para explicar que, gracias a esta, se justifica el progreso médico de los trasplantes de órganos teniendo como donadores a pacientes con muerte encefálica. Pero no deja claro a qué se refiere con ficción moral, puesto que primero afirmó que consistía en pensar que ante una situación de coma irreversible en la que el paciente aún está vivo, el acto de permitir que la muerte se produzca es un acto pasivo; por otro lado, afirma que en realidad el acto de permitir que se dé la muerte, retirando el soporte vital, causaría dicha muerte. Miller señala que, de acuerdo con esta perspectiva, retirar el tratamiento de mantenimiento a la vida es una omisión pasiva que solo permite que el paciente muera y, por consiguiente, equivale a una "ficción moral". Según lo anterior, podemos afirmar que la ficción moral se da en la propuesta de Jonas y no en la del Comité de Harvard, como afirma unas líneas antes, puesto que la ficción de Jonas se daría en la distinción que hace entre detener el soporte vital de vida, lo que posibilita que el paciente muera y, a su vez, causa la muerte al extraer los órganos.

Por lo anterior, la ficción moral según Miller no consiste en considerar que la muerte cerebral es la muerte del ser humano, en todo caso hace referencia al deber del médico de preservar la vida y no causar la muerte de sus pacientes; deber que defienden Jonas y el Comité de Harvard. Ahora bien, Jonas y el Comité de Harvard coinciden en que los médicos no deben matar a sus pacientes, pero difieren en lo que atañe a considerar a los pacientes con muerte encefálica como vivos. Mientras que Jonas y Miller sostienen que podrían estar vivos y que la extracción de órganos vitales causaría su muerte, El Comité de Harvard considera que están muertos de hecho. Por esta razón, Jonas concluye que la práctica de la extracción de órganos es incorrecta y debe cesar. Por el contrario, Miller considera que, aunque podrían estar vivos, la práctica no debe cesar, porque no está de acuerdo con la premisa de que "los médicos no deben matar a los pacientes". 
En suma, la ficción moral a la que se refiere Miller consiste en considerar que los médicos no deben matar a los pacientes, puesto que, si fuera tomado como cierto, no se podrían detener los respiradores artificiales al ser este acto también una causa de la muerte. Entonces, la ficción moral la cometen tanto el Comité de Harvard como Jonas, en el sentido en que no les parece correcto que el médico mate a la persona en coma irreversible y, por tanto, deciden crear la definición de muerte encefálica para justificar la extracción de órganos, a excepción de Jonas, quien se niega a dicha práctica.

Por ahora, a partir del razonamiento anterior, se concluye que Miller y Jonas dudan de la muerte de hecho del individuo en coma irreversible, pero difieren en la legitimidad de la extracción de órganos de tales individuos: mientras que Jonas niega tal legitimidad porque se provocaría la muerte del individuo, Miller asegura que sí se provocaría la muerte, pero se debe dudar de que el médico no deba causar la muerte del paciente, porque si fuera así, entonces tampoco deberíamos retirar el soporte vital. Frente a esta posición, se sostiene que "El individuo no fallece por la retirada de la reanimación, sino justamente a la inversa, se interrumpe la asistencia reanimadora porque el individuo ha fallecido" (34, p3). En otras palabras, se admite la muerte encefálica como un hecho, por lo que es necesario revisar la pertinencia de los criterios para su diagnóstico.

\section{Los criterios para diagnosticar la muerte encefálica}

Las pruebas para diagnosticar la muerte encefálica dieron como resultado otros criterios de muerte, con el fin de entender cuáles eran las funciones fundamentales para afirmar que un individuo había perdido la vida. Tales criterios de muerte son: la muerte de todo el encéfalo, la muerte del tronco encefálico y la muerte de la neocorteza cerebral. A continuación, se presenta el test expuesto en el Informe de Harvard basado en el criterio de todo el encéfalo y las críticas hechas al test para mostrar el desarrollo de los criterios del tronco encefálico y de la neocorteza cerebral. De acuerdo con el Comité de Harvard de 1938 (37, p337), estas son las características del coma irreversible:
1) Falta de receptibilidad y respuesta (estímulos externos).

2) No movimientos y respiración (cubrir un periodo de observación de por lo menos una hora para establecer la ausencia de espontaneidad, apagar el respirador mecánico por 3 minutos y observar si hay algún esfuerzo por parte del sujeto por respirar espontáneamente).

3) No reflejos o ausencia de reflejos provocados: pupila fija, dilatada y no responde a fuente de luz directa brillante; no hay movimiento ocular (al girar la cabeza o irrigar las orejas con agua helada); parpadeo ausente; no hay evidencia de actividad postural; suspensión de la deglución, bostezo y vocalización; reflejos corneales y faríngeos ausentes.

4) Electroencefalograma plano (prueba confirmatoria de 1, 2 y 3): excluir presencia de hipotermia (temperatura debajo de los $32.2 \mathrm{C}$ ) o de depresores del sistema nervioso central como barbitúricos.

El criterio de muerte de todo el encéfalo se refiere al cese de todas las estructuras intracraneales: hemisferios cerebrales, tronco encefálico y cerebelo (10, p100). Una de las críticas más fuertes a este criterio consiste en que hay pacientes que no cumplen las especificaciones como el cese completo e irreversible de todas las funciones del cerebro, puesto que ciertas actividades hormonales continúan dándose en pacientes diagnosticados con muerte encefálica (ME), como es el caso de la secreción de la arginina vasopresina (ADH), la cual es una hormona antidiurética que regula el funcionamiento renal. La ADH se sintetiza en neuronas localizadas en dos núcleos hipotalámicos (paraventricular y supraóptico) cuyos axones llegan a la hipófisis posterior o neurohipófisis y, al no regular dicha secreción, conduce a la aparición de diabetes insípida. La diabetes insípida (DI) es una enfermedad infrecuente producida por la falta absoluta o relativa de secreción o de acción de la ADH, con la consecuente poliuria por eliminación de un gran volumen de orina diluida. 
En algunos estudios se ha comprobado que hay pacientes diagnosticados con ME que no presentan diabetes insípida; en dado caso, si se quiere ser coherente con el criterio de muerte de todo el encéfalo, se debe afirmar que dichos pacientes siguen vivos.

La segunda parte de la argumentación afirma que esta función de regulación no es una simple actividad metabólica, sino una función del organismo como un todo:

$\mathrm{Y}$, segundo, porque esta regulación representa claramente una "función" y no la mera actividad metabólica de un grupo aislado de células, se trata de una actividad cerebral clínicamente observable (mantenimiento de una homeostasis correcta del agua y de los electrolitos corporales) y dirigida a mantener el funcionamiento del organismo como un todo $(7, \mathrm{p} 101)$.

El segundo argumento tiene que ver con la prueba del electroencefalograma. Aunque esta prueba es meramente confirmatoria, se recomienda su realización. Sin embargo, se han presentado casos en que los pacientes con muerte encefálica muestran tener actividad eléctrica, aunque puede ser considerada como no significativa para el funcionamiento del organismo como un todo; aun así, es controversial y en estricto sentido no es compatible con el criterio del cese completo e irreversible de todas las funciones del cerebro entero. En este mismo sentido, en un estudio reciente, coordinado por Taborda et al. (34, pp106-108), se mostró que, en algunas circunstancias, como la muerte asociada a la destrucción globo ocular, persiste el llenado de vasos intracraneales en algunas arterias, lo que puede conducir al diagnóstico de falsos positivos, a pesar de cumplir con el resto de los parámetros diagnósticos. De esta manera, surge el segundo criterio de muerte del tronco encefálico con el cual se especifica que la muerte es representada por la pérdida irreversible de la función del tronco encefálico. Este criterio deja de lado los hemisferios cerebrales y se centra solo en el tronco encefálico (o tallo cerebral), para hacer caso omiso a la objeción sobre la actividad de secreción de algunas hormonas y sobre la actividad eléctrica. A continuación, se presenta el test conocido como los "Criterios de Minnesota", propuesto por los neurocirujanos Mohandas y Chow (10, p99):
1) Lesión intracraneal conocida e irreparable:

- Ausencia de movimientos espontáneos

- Apnea (4 minutos)

- Ausencia de reflejos troncoencefálicos:

Pupilar

Corneal

Ciloespinal

Oculovestibular

Oculoencefálicos

Nauseoso

2) Hallazgos invariables durante al menos 12 horas

3) EEG no obligatorio

Según los anteriores criterios, la prueba del electroencefalograma no es obligatoria debido a que para el criterio del tronco encefálico no es importante la actividad eléctrica ni la regulación de hormonas. Por tanto, el énfasis de este criterio es la capacidad para respirar y la capacidad de conciencia. Una de las críticas a este criterio es la mencionada por Hans Jonas, la pérdida de la espontaneidad de la respiración, pues lo que se pierde es tal espontaneidad, pero el organismo puede seguir respirando así sea artificialmente. En este orden de ideas, la función de respirar que viene del tronco encefálico puede ser sustituida mecánicamente, por lo que no se puede considerar a alguien que no respira espontáneamente como muerto de hecho, independientemente de que tal respiración se dé artificialmente.

La segunda crítica se basa en la conciencia. Al respecto, Escalante (10) introduce la diferencia entre la capacidad para la conciencia y el contenido de la conciencia. Así mismo, afirma que es posible lograr un cierto grado de despertar al aplicar estímulos eléctricos, por lo que considera que perder la capacidad para la conciencia no es tan esencial al momento de diagnosticar a un individuo muerto de hecho.

El tercer criterio es el de muerte de la neocorteza cerebral, el cual se aplica a los individuos en estado vegetativo persistente y plantea que "la pérdida irreversible de las funciones corticales superiores, la conciencia y las funciones cognitivas son necesarias y suficientes para diagnosticar la muerte". 
(10, p102). Díaz Águila (35, p36), quien está a favor de esta posición, afirma que no se conoce el caso de un paciente que, ante el diagnóstico de coma, ausencia de reflejos del tallo cerebral y apnea, haya recuperado las funciones neurológicas.

Con este criterio toman relevancia los hemisferios cerebrales y pasan a un segundo plano las funciones de respiración y circulación. Una persona que pierde la conciencia pero que sigue respirando y su circulación sigue activa es una persona muerta de hecho. Este criterio es interesante porque enfatiza en la falta de conciencia para afirmar que se ha perdido la vida de la persona. Sin embargo, este criterio es polémico, dado que el criterio de muerte encefálica se ha pensado basado en cuestiones fisiológicas y, en cambio, el que nos ocupa está basado en cuestiones psicológicas. Además, las funciones cognitivas son difíciles de explorar, no se cuenta con un test para confirmar el diagnóstico de estado vegetativo persistente y tampoco se tiene certeza del significado del "contenido de la conciencia" como para asegurar que está ausente o hablar de su irreversibilidad. Por último, los problemas prácticos de este criterio pueden ser muchos dado que estamos frente a un ser que respira y mantiene su circulación espontánea y, no menos importante, mantiene sus reflejos (tiene la capacidad de toser, tener náuseas y parpadear) $(10$, p102).

Como ya se mencionó anteriormente, este criterio es polémico, puesto que no solo se basa en aspectos fisiológicos, sino psicológicos y desafía aun más la visión que tiene la sociedad acerca de la muerte del ser humano. En un sentido análogo, Ari Joffe plantea que no existe un criterio confiable para diagnosticar lo que nos hace humanos, por lo que tampoco podríamos saber el momento en que se han suspendido estas cualidades. Diagnosticar con dichos criterios la muerte nos conduciría a "numerosos problemas teóricos y prácticos, entre ellos admitir que un individuo podría ser declarado muerto incluso antes de que su cuerpo estuviera muerto". Dicha concepción la sustenta en el reconocimiento de funciones que no dependen de la actividad cerebral (41, p489).

Actualmente, nos encontramos ante un problema ético de la medicina: la evolución de los argumentos en los criterios de muerte basados en la definición de muerte encefálica se pone en duda por diversos casos en que individuos en coma irreversible no satisfacen los criterios ni los test con los que son valorados. Con lo anterior, nos referimos a casos en que no se pierden todas las funciones del encéfalo (secreción de hormonas de la hipófisis y el hipotálamo), la capacidad de conciencia y las funciones vegetativas de respiración y circulación espontánea.

Con el fin de entender mejor la necesidad de la evolución histórica de los criterios de muerte, presentamos la visión de Eduardo Rivera López acerca de la muerte y "el paradigma biológico", retomada por Bernat, Gert y Culvert (38). Tal visión se opone a la de John P. Lizza, quien en su texto Persons, humanity and definition of the death (18) afirma que la muerte es una cuestión más metafísica, cultural y social que biológica. Por su parte, Rivera distingue en su texto tres instancias para declarar la muerte: la definición de la muerte, el criterio o los criterios de muerte (condiciones necesarias y suficientes) y los test utilizados para verificar el criterio que satisface la definición de muerte. Esta idea la representamos en el siguiente recuadro (Figura 1):

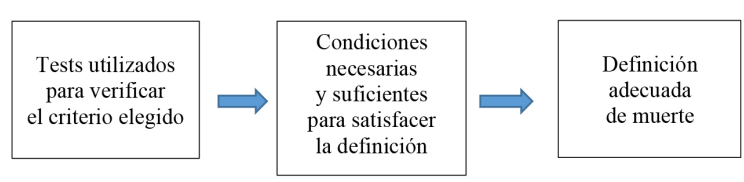

Figura 1. Instancias para declarar la muerte Fuente: elaboración propia.

Según Rivera, las preguntas a plantear son: ¿qué características deberían incluirse en una definición de muerte para que sea adecuada?, ¿cuáles son las condiciones necesarias y suficientes que satisfacen la definición adecuada de la muerte? y ¿los test utilizados verifican el criterio de muerte elegido? A la pregunta sobre las características que debería tener la definición de muerte responde:

1) La definición de muerte no debe ser meramente estipulativa.

2) El concepto de muerte debe ser fundamentalmente biológico.

3) El concepto debe tener la idea de irreversibilidad.

4) La muerte debe considerarse un evento instantáneo, no un proceso. 
Bernat propone como definición de muerte "el cese irreversible de todas las funciones críticas del organismo como un todo". Ahora bien, ¿¿cuáles son esas funciones críticas? Al respecto, Rivera menciona tres:

1) Las funciones de respiración espontánea y control autónomo de la circulación.

2) Las funciones integrativas que permiten la homeostasis del organismo.

3) La conciencia, en la medida en que es necesaria para responder a los requerimientos de la hidratación, nutrición y protección $(27, \mathrm{p} 40)$.

Esta definición es importante puesto que las legislaciones de la mayoría de los países asientan sus bases en ella para establecer la muerte encefálica como muerte de hecho del individuo (Tabla 1) (8, p189):

Tabla 1. Muerte encefálica: diagnóstico clínico y pruebas instrumentales

Muerte encefálica: diagnóstico clínico y pruebas instrumentales

DIAGNÓSTICO CLÍNICO

1

Coma estructural, de etiología conocida y carácter irreversible.

Condiciones clínicas generales durante la exploración neurológica. Estabilidad cardiocirculatoria:

2 Oxigenación y ventilación adecuada Ausencia de hipotermia $<32^{\circ} \mathrm{C}$ Ausencia de enfermedades metabólicas importantes Ausencia de tóxicos o fármacos depresores del SNC

Exploración clínica neurológica. Exploración neurológica cerebral: coma arreactivo

3 Exploración neurológica troncoencefálica: ausencia de reflejo fotomotor, reflejo corneal, reflejo oculocefálico, reflejo oculovestibular, reflejo nauseoso, reflejo tusígeno, respiración espontánea y respuesta al test de atropina.

4 Puede haber actividad motriz de origen modular, tanto refleja como espontánea.

5 Periodo de observación: 6 h. Modificable a criterio medico según tipo y gravedad de la lesión causal.

En las lesiones de localización infratentorial:

6 exploración clínica más EEG y/o un método diagnóstico instrumental que confirme la ausencia de función de los hemisferios cerebrales.
PRUEBAS INSTRUMENTALES

Electrofisiológicas: electroencefalograma, Bispectral Endex

1 Scale y potenciales evocados auditivos y somatosensoriales de nervio mediano.

Valoran la circulación cerebral:

Sonografía Doppler transcraneal

Arteriografía cerebral de cuatro vasos

2 Arteriografía cerebral por sustracción digital

(arterial o venosa)

Angiografía cerebral mediante Tc multicorte

Angiogammagrafia cerebral con 99Tc-HMPAO u otros

radiofármacos difusibles

Fuente: Escudero $(8,2009)$.

De igual forma, en las Conclusiones de la III Conferencia de Consenso de la semicyuc y Muerte encefálica en las Unidades de Cuidados Intensivos se establece la muerte como el cese irreversible de las funciones de las estructuras neurológicas intracraneales (hemisferios cerebrales, tronco encéfalo y cerebelo), es decir, como único criterio aceptado para la muerte de todo el encéfalo, lo cual excluye los criterios de muerte del tronco encefálico y el de la neocorteza cerebral. Otras definiciones, tales como "muerte troncoencefálica" y "muerte de la neocorteza" (estado vegetativo persistente, anencefalia) no cumplen los criterios de la definición establecida en esta conferencia. (7, p195)

La Red/Consejo Iberoamericano de Donación y Trasplante (RCIDT) está compuesta por 21 países (España, Portugal, Brasil y todos los países americanos de habla hispana) y fue creada en Granada (España) en la VIII Conferencia Iberoamericana de Ministros de Salud, el septiembre del año 2005. Dicha red realizó un estudio sobre los criterios de diagnóstico de la muerte encefálica, en donde especifica, en primera instancia, que el diagnóstico de muerte encefálica se basa en la demostración de un coma arreactivo, ausencia de reflejos troncoencefálicos y ausencia de respiración espontánea; segundo, que la muerte encefálica se considera la muerte del individuo (excepto en Nicaragua porque, aunque pertenezca a la (RCIDT), no tiene legislación específica) y, por último, que existen diferencias importantes entre los 21 países que conforman la red, tales como los prerrequisitos clínicos, la exploración neurológica, el tiempo de observación, los test de diagnóstico instrumentales y las decisiones tras el diagnóstico de ME. 
Podemos notar que los criterios actuales están basados en el criterio de todo el encéfalo que proponía Bernat; sin embargo, cabe resaltar que aún es vigente la crítica acerca de la persistencia de las funciones hormonales y de la pérdida de conciencia. Esta postura sobre la muerte de todo el encéfalo excluye todo el espectro de la conciencia del ser humano y reduce la muerte humana a cuestiones biológicas, sin tener en cuenta que también implica nociones ontológicas y sociales. En otras palabras, esta postura deja fuera a las personas en estado vegetativo persistente porque no se basa en el concepto de persona que especifica Lizza. En la misma línea de razonamiento, Tobias Eichinger, en su texto Brain Death, Justified Killing and the Zombification of Humans - Does the Transplantation Dilemma Require New Ways of Conceptualizing Life and Death?, sostiene que considerar la ME como muerte de hecho no es una cuestión meramente técnica, por lo que se hace necesario hacer dos preguntas cuya naturaleza de respuesta no es la misma: ¿el paciente tiene muerte encefálica? y ¿el paciente con muerte encefálica está muerto de hecho? La respuesta a la primera pregunta es de naturaleza técnica y neurológica, mientras que la segunda pregunta implica consideraciones filosóficas, antropológicas y ontológicas $(6, \mathrm{p} 12)$. Por esta razón, podemos advertir que existen actualmente diversos informes internacionales que son aceptados como recomendaciones oficiales de diagnóstico de la muerte encefálica e igualmente informes sobre las pruebas clínicas e instrumentales recomendadas para diagnosticar la ME. No obstante, de acuerdo a lo reportado en el estudio de la Red Iberoamericana, actualmente no se siguen tales recomendaciones de manera homogénea en los 21 países que conforman la red. Además de la falta de homogeneidad, Barreto et al., (36, pp35-40) ha evidenciado en un estudio reciente la falta de capacitación entre los médicos en actualización sobre los criterios diagnósticos de la ME. Allí destaca lo preocupante que puede ser la falta de homogeneidad en los criterios de diagnóstico, la cual también se presenta en los países europeos, como lo afirman Thomas Bein, Müller y Citerio (39).

Una razón de esta inconsistencia en la práctica de diagnóstico y decisiones sobre la muerte encefálica es la dificultad de legislar de manera homogénea en cada uno de los países que integran la red. Mientras que unos países sí especifican en sus reglamentos retirar el soporte vital una vez declarada la muerte encefálica, otros no lo hacen y, por tanto, mantienen los soportes activos. Por su parte, los prerrequisitos clínicos también presentan diferencias, por ejemplo, la temperatura mínima recomendada para descartar hipotermia es de $32.2^{\circ} \mathrm{C}$, pero en algunos países se exige una temperatura mínima de $35^{\circ} \mathrm{C}$ sin justificación alguna. Adicionalmente, es importante tener cuidado al revisar las distintas causas del coma, puesto que el nivel o contenido de la consciencia puede ser conflictivo si no se realiza un adecuado test para determinar la causa del coma; lo que lleva en ocasiones a errores médicos graves (40). Ahora bien, estas diferencias en el diagnóstico y en los test para constatar la $\mathrm{ME}$ nos muestran la gran dificultad que existe al llevar una práctica médica homogénea, lo que implica que la toma de decisiones sea más complicada en su realización. Es aquí donde entran los problemas éticos sobre la extracción de órganos y el retiro del soporte vital: si no es posible realizar un adecuado test para diagnosticar la muerte de un individuo, ¿cómo podemos realizar un adecuado retiro del soporte vital o una adecuada extracción de órganos?

Por su parte, Lizza está en contra del paradigma biológico porque afirma que es un enfoque materialmente reduccionista de la personalidad y la humanidad. Dicho enfoque se basa en la creencia de que los organismos no son más que agregados de sustancias. En esta investigación se refiere a reducir al ser humano a conceptos que solo implican cuestiones fisiológicas (18, p253). Su crítica va fundamentalmente en contra de la noción de muerte basada en el criterio neurológico que concibe al encéfalo como el integrador del organismo como un todo. Esta noción de muerte considera que, aunque la pérdida de todas las funciones cerebrales lleve a la pérdida de la consciencia, perder la consciencia no es significativo para la pérdida del ser humano o persona. Lo anterior implica que las personas en estado vegetativo permanente (EVP) no sean consideradas como muertas, porque al continuar el funcionamiento de su tronco encefálico, continúa su integración orgánica. El autor está en contra de 
considerar que la definición de muerte debe ser estrictamente unívoca para todos los organismos, es decir, construirse de acuerdo al tipo de cosa que muere, por tanto, al definir la muerte del ser humano debe tomarse en cuenta lo que el ser humano es como tal.

La crítica que hace Lizza a los defensores del paradigma biológico, es decir, a Bernat, Culver y Gert (39, pp389-394), apunta al hecho de no considerar a la personalidad como fundamental para la pérdida de la vida del ser humano. En su definición, afirman que la muerte es el cese permanente del funcionamiento del organismo como un todo. En cambio, Gert es más específico y afirma que "Un organismo está muerto si y solo si 1) ha dejado de funcionar permanentemente como un todo y, cuando corresponda, 2) ha perdido permanentemente la conciencia, 3) todas las partes identificables del organismo han perdido permanentemente la conciencia" (18, p23). En su artículo "A complete definition of death" (38, p389), Gert afirma lo siguiente: "Cuando la cabeza cortada de un mono responde a los sonidos y visiones, afirmar que está muerto altera la comprensión ordinaria de la muerte mucho más que afirmar que el mono todavía está vivo". Lo que quiere decir que el hecho de que la cabeza continúe respondiendo a los sonidos y visiones permitiría sostener la idea de que la cabeza es una parte identificable del organismo en la cual no hay pérdida de consciencia. El punto tres se entiende a la luz de que Gert considera que el cese de las funciones del cerebro superior no es muerte en sí, sino que es necesario que se considere en conjunto con los puntos 1 y 2 . Al respecto, Lizza afirma que Gert sí toma en cuenta la pérdida irreversible de la conciencia para definir la muerte, pero en conjunto con la pérdida de la integración orgánica, es decir, Gert no acepta que la pérdida de la conciencia por sí sola sea la muerte de la persona. El problema con esta postura es que ve la pérdida de la conciencia como fundamental para definir la muerte, pero en conjunto con la pérdida del funcionamiento del organismo como un todo. El problema, según el autor, es que Gert no ve a la conciencia como parte fundamental del ser humano. En suma, según Lizza, se trata de encontrar las condiciones necesarias y suficientes para establecer lo que es una persona, porque dependiendo de estas condiciones se entenderá cuáles y qué tipo de cosas entran dentro de este concepto.

Un aspecto adicional para analizar el fenómeno de la muerte ha sido planteado desde las ciencias sociales $y$, aunque no es el objetivo central de este estudio, sí conviene destacar desde esta perspectiva el carácter instrumental con que se designa y diagnostica la muerte. De acuerdo con Norma González, por medio de la ciencia y la técnica, aunado al prestigio que se les concede en las sociedades contemporáneas, es posible la admisión de visiones sobre la vida, la salud y la muerte. Así, "La medicina forma parte privilegiada de esos mecanismos y estrategias de saber/poder a partir de los cuales se puede regular y administrar la conducta de las poblaciones" (12). En este sentido, la autora sostiene que el discurso médico sobre la muerte constituye el punto de vista de una disciplina entre otras subjetividades y en la manera de asimilar la muerte. En otras palabras, los discursos sobre la muerte habrían de considerar no solo aspectos de orden biomédico, sino también aspectos propios de la cultura y la historia como constituyentes esenciales para comprender la salud la vida y la muerte (12).

\section{Conclusiones}

Retomando las cuestiones al inicio de este artículo, podemos concluir que la aceptación de la definición de muerte encefálica como muerte continúa generando conflictos, especialmente por la falta de certeza para poder definir la muerte, además de las interrogantes morales que se generan al realizar otro tipo de acciones, tales como usar los órganos para trasplante o desconectar a un paciente cuyas funciones cerebrales no manifiestan actividad.

Por lo anterior, ante la falta de consensos en su definición, cualquier estrategia diagnóstica que se postule para afirmar que un individuo con muerte encefálica está muerto de hecho, será motivo de controversias o refutaciones.

A pesar de lo anterior, los motivos para postular la muerte encefálica como muerte tienen sentido, ya que la posibilidad de ofrecer una alternativa de vida a partir de un trasplante es una acción técnica valorada positivamente, así como aquellas 
alternativas terapéuticas que tienen como fin aliviar la enfermedad y evitar una muerte prematura. Del mismo modo, evitar el ensañamiento terapéutico de un paciente que tiene nulas posibilidades de sobrevida, mediante la eliminación del soporte artificial, también es considerado como un acto altruista al contribuir al bien morir. Finalmente, el respeto a la voluntad de los pacientes a morir o admitir la muerte encefálica como muerte parece ser otra razón suficiente para evitar prolongar los soportes que apoyan artificialmente al mantenimiento de la vida.

Las diferencias en torno a la definición de la muerte encefálica como muerte de hecho son vigentes y conviene que la investigación continúe desde diferentes perspectivas ya que, como se ha hecho notar, la muerte no es un tema que involucre solo aspectos fisiológicos, por tanto, la búsqueda de acuerdos entre los profesionales y ciudadanos involucrados en la disputa podría contribuir a tener mayor claridad en la comprensión de la muerte, así como mejorar las acciones terapéuticas, las normas y las incertidumbres morales de los individuos.

\section{Referencias}

1. Abbagnano N. Diccionario de filosofía. Segunda edición. México: FCE; 1974.

2. Aristóteles. Acerca del alma. Primera edición. Martínez TC, editor. Madrid: Gredos; 1978.

3. Becchi P. Muerte cerebral y trasplante de órganos. Un problema de ética jurídica. Primera edición. Madrid: Editorial Trotta; 2007.

4. Bromberger S. On what we know we don't know. Explanation, theory, linguistics, and how questions shape them. Press TUoC, ed. Chicago; 1992. Disponible en: https://web.stanford.edu/group/cslipublications/ cslipublications/bromberger-corpus/On-What-WeKnow-We-Dont-Know.pdf

5. Casati, RO, Varzi, AC. Events. In: The Stanford encyclopedia of philosophy. Zalta EN, editor; 2010.

6. Eichinger T. Brain death, justified killing and the zombification of humans - Does the transplantation dilemma require new ways of conceptualizing life and death? In: Jox R, Assadi G, Marckmann G, eds. Organ transplantation in times of donor shortage. International library of ethics, law, and the new medicine, vol 59. Springer, Cham; 2017. https://doi.org/10.1007/9783-319-16441-0_2
7. Escalante JL, Escudero D. Conclusiones de la III Conferencia de consenso de la SEMICyuc, Muerte encefálica en las Unidades de Cuidados Intensivos. Medicina Intensiva 2000;24(4): 193-97.

8. Escudero D. Diagnóstico de muerte encefálica. Medicina Intensiva. Hospital Universitario Central de Asturias. Oviedo. 2009; 33(4):185-95. https://doi. org/10.1016/S0210-5691(09)71215-X

9. Escudero D. Muerte encefálica en Iberoamérica. Medicina Intensiva. 2009; 33 (9): 415-423. https://doi.org/10.1016/j.medin.2009.07.011

10. Escalante JL. Muerte encefálica. Evolución histórica y situación actual. Medicina Intensiva. 2000; 24 (3). https://doi.org/10.1016/S0210-5691(00)79569-6

11. García-García E. Diabetes insípida. Endocrinología Pediátrica. Hospitales Universitarios Virgen del Rocío. 2011; 1(1).

12. González-González N. Medicalización de la muerte. Elementos de discusión y análisis para un abordaje crítico desde las Ciencias Sociales. Revista Culturales. 2005; 6(1): 1-27. https://doi.org/10.22234/ recu.20180601.e350

13. García-Porrero JA, Hurlé JM. Neuroanatomía humana. España: Editorial Médica Panamericana SA; 2015.

14. Granero J, Fernández C, eds. Soporte vital básico y avanzado. Basado en las recomendaciones ERC-2010. Almería; 2011. Disponible en: https://core.ac.uk/ download/pdf/143457138.pdf

15. Humphry, D, Wickett, A. El derecho a morir. Comprender la eutanasia. España: Tusquets Editores; 2005.

16. 16. Jonas H. Técnica, medicina y ética. Primera Edición. España: Paidós; 1997.

17. Diario Oficial de la Federación. Ley General de Salud. México; 2018.

18. Lizza JP. Persons, humanity and the definition of the death. Baltimore: The Johns Hopkins University Press; 2006.

19. Miller FG. Death and organ donation. J. Med Ethics. 2009; 35. https://doi.org/10.1136/jme.2009.030627

20. Monzón JL. Recomendaciones de tratamiento al final de la vida del paciente crítico. Med Intensiva. 2008; 32(3). https://doi.org/10.1016/S0210-5691(08)70922-7

21. Viniegra VL. El reduccionismo científico y el control de las conciencias. Parte I. Boletín Médico del Hospital Infantil de México. 2014; 71(4). https://doi.org/10.1016/j.bmhimx.2014.05.001

22. Souza, J.P., Oliveira-Neto, A., Surita, F.G. et al. The prolongation of somatic support in a pregnant woman with brain-death: a case report. Reprod Health 3, 3 (2006). https://doi.org/10.1186/1742-4755-3-3 
23. Singer P. Repensar la vida y la muerte. El derrumbe de nuestra ética tradicional. Barcelona: Editorial Paidós; 1997.

24. Shewmon DA. Chronic "brain death": Meta-analysis and conceptual consequences. American Academy of Neurology. 2009 Septiembre; 7 (51). https://doi. org/10.1212/WNL.51.6.1538

25. Secretaría de Salud. Programa de acción: trasplantes. México; 2001.

26. Rodríguez D. Muerte cerebral y trasplante de órganos. Aspectos internacionales y éticos. España: Universidad de Salamanca; 2008.

27. Rivera-López E. Ética y trasplante de órganos. México: Fondo de Cultura Económica; 2001.

28. Platón. Fedón. Diálogos III. España: Editorial Gredos; 1988.

29. Tamayo RP. La construcción de la bioética. Vol. 1. México: Fondo de Cultura Económica; 2008.

30. Pernick M. Death: beyond whole-brain criteria. Publishers KA, editor. US: Springer; 1988.

31. Moreno-Ruiz N. Mora-Pabón G. Heparina no fraccionada en el manejo del síndrome coronario agudo. Rev Fac Med [Internet]. 2007 Mar; 55( 1 ): 43-57.

32. Monzón JL. Recomendaciones de tratamiento al final de la vida del paciente crítico. Medicina Intensiva. 2008; 32(3). https://doi.org/10.1016/S0210-5691(08)70922-7

33. Martin-Fumadó C, Gómez-Durán E, Galán-Serrano J, Barbería-Marcalaina E, Arimany-Manso J, Parera-Ruize A. Toma de decisiones al final de la vida y ante el diagnóstico de muerte encefálica. Revista Española de Medicina Legal. 2017; 43(3). https://doi.org/10.1016/j.reml.2017.05.003

34. Taborda M, Coalla M, Lépore N, Sacco P, Grottasanta S, Corchuelo D, Braida N. Disociación clínico-imagenológica en determinación de muerte encefálica. Neurología Argentina. 2019; 11(2). https://doi.org/10.1016/j. neuarg.2019.02.005

35. Díaz-Águila H. Muerte encefálica. Rev Cuba Med Int Emerg [revista en Internet]. 2018; 17(0). Disponible en: http://www.revmie.sld.cu/index.php/mie/article/ view/444

36. Barreto V, Unigarro L, Del Pozo G. ¿Cuánto conocen los médicos sobre el diagnóstico y certificación de muerte encefálica? Rev Fac Cien Med (Quito). 2017; 42(2).

37. A definition of irreversible coma: report of the Ad Hoc Committee of the Harvard Medical School to examine the definition of brain death. JAMA. 1968 Aug 5; 205(6): 337-40.

38. Bernat JL, Culver CM, Gert B. On the definition and criterion of death. Ann Intern Med. 1981
Mar;94(3):389-94. doi: 10.7326/0003-4819-94-3-389. PMID: 7224389.

39. Bein T, Müller T, Citerio G. Determination of brain death under extracorporeal life support. Intensive Care Med. 2019 Mar;45(3):364-366. https://doi. org/10.1007/s00134-018-05510-z

40. Rabinstein AA. Coma and Brain Death. Continuum (Minneap Minn). 2018 Dec;24(6):1708-1731. https:// doi.org/10.1212/CON.0000000000000666

41. Moraes-Rodrigues F, Junges JR. Morte encefálica: uma discussão encerrada?. Revista Bioética [Internet]. 2015;23(3):485-494. https://doi.org/10.1590/198380422015233085

42. Rodríguez D. Muerte cerebral y trasplante de órganos. Aspectos internacionales y éticos. Universidad de Salamanca, España; 2008. 